\title{
ESSENTIAL SPECTRA OF SOME MATRIX OPERATORS BY MEANS OF MEASURES OF WEAK NONCOMPACTNESS
}

\author{
Boulbeba Abdelmoumen
}

Abstract. In this paper, we give some results concerning stability in the Fredholm theory via the concept of measures of weak noncompactness. These results are exploited to investigate the essential spectra of some matrix operators on Banach spaces. This work contains some results which extend some well known ones in the literature.

Mathematics subject classification (2010): 28A78, 47A10, 47A53, 47A55, 47N20.

Keywords and phrases: Measures of weak noncompactness in Banach spaces, Fredholm operators, essential spectra.

\section{REFERENCES}

[1] B. Abdelmoumen, Essential Spectra on Spaces With the Dunford-Pettis Property and Application to Transport Operators, Indi. J. Pure. Appl. Math, (IJPA-D-12-00898), (2012).

[2] B. Abdelmoumen, A. Dehici, A. Jeribi And M. Mnif, Some New Properties of Fredholm theory, essential spectra and application to transport theory, J. Ineq. Appl., (2008), 1-14.

[3] B. Abdelmoumen And H. Baklouti, Perturbation results on semi-Fredholm operators and applications, J. Ineq. Appl., (2009), Article ID 284526, 13 pages doi:10.1155/2009/284526.

[4] B. Abdelmoumen, A. JeRIBI AND M. MNIF, On graph measures in Banach spaces and description of essential spectra of multidimensional transport equation, Acta. Math. Scie. Acta Math Scie, 32B (5) (2012), 2050-2064.

[5] F. V. Atkinson, H. Langer, R. Mennicken and A. Shkalikov, The essential spectrum of some matrix operators, Math. Nachr., 167 (1994), 5-20.

[6] J. Banaś And Rivero, On measures of weak noncompactness, Ann. Mat. Pura Appl, 151 (1988), 213-262.

[7] J. Diestel, A survey of results related to Dunford-Pettis property, Contemp. Math. 2 (1980), 15-60.

[8] N. Dunford And B. J. Pettis, Linear operations on summable functions, Trans. Amer. Math. Soc., 47 (1940), 323-392.

[9] N. Dunford, J. T. SchwartZ, Linear operators, Part I: General theory. Interscience Publishers, Inc., New York, (1958).

[10] A. Grothendieck, Sur les applications linéaires faiblement compactes d'espaces du type $C(K)$, Canad. J. Math., 5 (1953), 129-173.

[11] A. Jeribi AND M. MNIF, Fredholm Operators, Essential Spectra and Application to Transport Equations, Acta. Appl. Math., 89 (2005), 155-176.

[12] T. Kato, Perturbation Theory for Linear Operators, Springer-Verlag, New York, 1966.

[13] K. Latrach, Essential spectra on spaces with the Dunford-Pettis property, J. Math. Anal. Appl. 233 (1999), 607-622.

[14] N. Moalla, M. DAMAK AND A. JeRIBI, Essential spectra of some matrix operators and application to two-group transport operators with general boundary conditions, J. Math. Anal. Appl. 323 (2006), 1071-1090.

[15] V. MÜLLER, Spectral theory of linear operators and spectral systems in Banach algebras, Birkhäuser (2007).

[16] R. D. Nussbaum, Spectral mapping theorems and perturbation theorem for Browder's essential spectrum, Trans. Amer. Math. Soc., 150, 445-455 (1970). 
[17] M. SCHECHTER, Invariance of essential spectrum, Bull. Amer. Math. Soc., 71 (1965), 365-367.

[18] M. Schechter, Principles of Functional Analysis, Academic Press, New York, 1971.

[19] A. A. ShKalikov, On the essential spectrum of some matrix operators, Math. Notes, 58, 6 (1995), 1359-1362.

[20] F. WOLF, On the invariance of the essential spectrum under a change of the boundary conditions of partial differential operators, Indag. Math., 21 (1959), 142-147. 